\title{
Pour une presse spécialisée en éducation au
} Sénégal

Le paysage médiatique sénégalais

For a specialised educational press in Senegal

Para una prensa especializada en educación en Senegal

\section{Abdou Salam Sall}

\section{(2) OpenEdition}

\section{Journals}

Édition électronique

URL : http://journals.openedition.org/ries/4007

DOI : $10.4000 /$ ries.4007

ISSN : 2261-4265

\section{Éditeur}

Centre international d'études pédagogiques

\section{Édition imprimée}

Date de publication : 1 septembre 2014

Pagination : 93-98

ISBN : 978-2-85420-604-3

ISSN : 1254-4590

\section{Référence électronique}

Abdou Salam Sall, «Pour une presse spécialisée en éducation au Sénégal », Revue internationale d'éducation de Sèvres [En ligne], 66 | septembre 2014, mis en ligne le 01 septembre 2016, consulté le 23 juin 2020. URL : http://journals.openedition.org/ries/4007 ; DOI : https://doi.org/10.4000/ries.4007 


\title{
Pour une presse spécialisée en éducation au Sénégal ${ }^{\star}$
}

\section{Le paysage médiatique sénégalais}

\begin{abstract}
Abdou Salam Sall
La presse sénégalaise peut se targuer d'avoir beaucoup contribué aux deux alternances politiques récentes au Sénégal. Il s'agit principalement d'une presse écrite, même s'il existe des radios, des chaînes de télévision et des médias en ligne, et d'une presse événementielle plutôt que d'une presse d'investigation, notamment sur des sujets en économie ou en éducation. Elle traite dans une large mesure du sensationnel, des événements politiques, des faits divers, etc. Il existe très peu de rubriques éducation dans les rédactions. En outre, rares sont les journalistes ayant reçu une formation à leur métier, au sein d'une école. Les pigistes sont très nombreux, au point que les journalistes qui se regroupent actuellement en corporation n'acceptent que ceux qui ont bénéficié d'une formation.

Pour parler de l'éducation dans les médias, il faudrait d'abord être journaliste et ensuite être spécialiste de l'éducation.

Cela n'existe pas encore au Sénégal même si, pour compléter ce paysage rapidement brossé, il faut noter quelques initiatives isolées : ainsi, l'université Cheikh Anta Diop a disposé pendant quelques années d'un journal intitulé Interface et le Syndicat autonome de l'enseignement supérieur publie un journal de façon épisodique. Enfin, très récemment, un jeune promoteur d'une télévision éducative, une "télé-école ", a lancé de sa propre initiative sa télévision dédiée exclusivement à l'éducation. Certaines émissions sont animées par des élèves pour des élèves. Moi-même, je crée actuellement une radio communautaire à Podor, avec notamment des émissions animées par les élèves pour les élèves. Amadou Wade Diagne vient de lancer un journal dédié exclusivement à l'éducation : Réussir Education. Avec le développement des nouvelles technologies, les Assises de l'éducation vont certainement recommander la création d'une télévision éducative proposant des ressources pédagogiques. La création de médias dédiés exclusivement à l'éducation marquera une évolution importante au Sénégal et de façon plus générale dans le contexte africain. L'enjeu est évident : quand l'éducation est intériorisée par une société, on peut en attendre des effets bénéfiques.
\end{abstract}

\footnotetext{
* Cet article est issu d'un entretien entre Abdou Salam Sall, Xavier Pons et Marie-José Sanselme, le 10 juillet 2014. L'entretien a été transcrit par Sylvaine Herold et révisé par Marie-José Sanselme et Xavier Pons. (NdIR)
} 


\section{Une initiative individuelle récente}

Porté par un consultant spécialisé en éducation, le journal Réussir l'éducation a publié son premier numéro en mai 2014. On y trouve des articles sur des événements, des interviews d'anciens élèves ou étudiants, ainsi qu'une couverture des Assises de l'éducation et de la formation. Cette initiative, de même que la publication récente d'une recherche sociologique sur l'amélioration de la qualité du système éducatif sénégalais, prend place dans un contexte général de prise de conscience des besoins éducatifs au Sénégal et de la nécessité d'impliquer le grand public. L'éducation n'est pas la seule affaire des politiques ou des fonctionnaires. Les ressources humaines présentes dans le pays, en fonction de leurs capacités, peuvent proposer des éléments utiles au système, sur la base d'informations objectives. Les Assises de l'éducation soutiennent toutes les initiatives qui vont dans ce sens.

\section{LA PRESSE EN ÉDUCATION : UN RÔLE À REDÉFINIR}

Les articles publiés dans la presse sénégalaise en matière d'éducation portent le plus souvent sur des événements récurrents tels que la rentrée des classes, les résultats au concours général, des résultats ou sur des problèmes comme les grèves et les dérives des enseignants et des élèves. Il existe très peu d'articles de fond sur le système éducatif. Mais les journalistes ont-ils la qualification requise pour traiter ces sujets? Si l'on n'est pas spécialisé en éducation, peut-on comprendre les éléments qui déterminent la qualité d'un système éducatif ? Si la presse couvre en général les manifestations de l'éducation et les relaie, reçoit-elle suffisamment d'informations? Les acteurs de l'éducation sont-ils conscients de la place et du rôle de la presse pour mettre l'éducation au cœur de l'ensemble des stratégies?

Lorsque j'étais recteur de l'Université Cheikh Anta Diop (UCAD) de Dakar, j'avais créé une direction de la communication, car je me rendais compte que, quelles que soient les actions menées et les efforts entrepris, on ne parlait de l'université que lorsqu'il y avait une grève et qu'on avait tendance à oublier tout ce qui se faisait en matière de recherche, de bonnes pratiques, de bons fonctionnements, de pratiques innovantes. Donc il fallait une direction de la communication pour informer largement l'opinion. Si, dans le cadre des Assises de l'éducation, nous voulons aujourd'hui utiliser l'assurance qualité, il faudrait que des journalistes puissent consulter les divers documents, les grilles de performance des différents établissements, afin de publier des articles de fond qui permettraient au grand public de se faire une opinion sur ce qui va ou ne va pas, et sur les sujets importants.

Les articles publiés actuellement tablent souvent sur le goût du public sénégalais pour le sensationnel. Et il y a parfois une grande différence entre un titre provocateur et l'article lui-même. Cela fait vendre, dans un contexte culturel globalement peu élevé. Dans le champ de l'éducation, la presse sénégalaise n’a 
pas encore mesuré le rôle qu'elle pouvait jouer pour infléchir certaines façons d'organiser le système éducatif, de penser les politiques éducatives. La presse relaie ce lui est apporté mais les journalistes n’ont pas eux-mêmes les ressources nécessaires pour analyser cette information de façon critique, et apporter une plusvalue. En revanche, ils relaient sans difficulté les perturbations, les images négatives de l'éducation, sans prêter la même attention à ceux qui essaient de s'investir dans un environnement difficile et qui ne sont ni connus ni reconnus. Par exemple, nombre d'établissements scolaires au Sénégal sont le fruit des communautés, grâce à l'aide des Sénégalais expatriés. C’est encore largement ignoré.

\section{REFONDER L'ÉDUCATION AU SÉNÉGAL, UN ENJEU DE SOCIÉTÉ}

Au cour des Assises de l'éducation, il y a l'idée que l'éducation est l'alpha et l'oméga de toutes choses. Il faut faire partager par tous cette conviction. Cela commence, par exemple, par le rang protocolaire du ministre en charge de l'éducation. C'est toute une cohérence à mettre en place. Il faut que les questions d'éducation deviennent un sujet central, dont on discute, afin que se crée la tension positive qui permet d'avancer. Et pour cela, il faudrait pouvoir partager les données disponibles sur l'éducation, afin que le débat public soit alimenté non par du ressenti, mais par des faits.

Les Assises de l'éducation sont une initiative du gouvernement afin de refonder le système éducatif, à la demande de plusieurs corps sociaux, notamment les syndicats d'enseignants, la société civile et d'autres partenaires. Le gouvernement a mis en place des comités, chargés de proposer une synthèse de l'éducation héritée de la colonisation, des cinq siècles d'éducation islamique avant la colonisation et de l'éducation traditionnelle basée sur les contes, légendes, rites, proverbes, etc. Tous les grands érudits sénégalais sont issus de ce magma-là. Mais aujourd'hui, comment faire? Combien de Sénégalais comprennent le français, qui est pourtant langue d'enseignement? Nos langues nationales ne sont pas encore généralisées dans le système éducatif. Dans les territoires où l’on a commencé à introduire les langues nationales, les communautés se sont senties beaucoup plus concernées par l'école que les autres communautés. Dans certaines communautés, l'école fournit l'instruction mais l'éducation est religieuse. Il nous appartient de faire la synthèse de tout cela pour que notre système éducatif se conforme à nos valeurs sénégalaises d'ouverture.

Nous devons, tout en nous adossant aux valeurs de notre civilisation, nous ouvrir sur un monde changeant dominé, dans une large mesure, par la science et la technologie. Notre système éducatif actuel est totalement désintégré. Seuls $13 \%$ des enfants ont accès à l'école préscolaire, si l'on ne tient pas compte des enfants qui se trouvent à cet âge dans les écoles coraniques. Si le taux d'accès au primaire est relativement acceptable, les abandons précoces sont nombreux. 
Certaines familles considèrent que l'éducation doit être seulement religieuse. Le taux de transit du primaire au secondaire s'améliore mais n'atteint pas, et de loin, $100 \%$. Au secondaire, on constate un rétrécissement de l'accès et notamment un déséquilibre entre les filières scientifiques, qui accueillent $30 \%$ des apprenants, contre $70 \%$ pour les filières littéraires. La parité filles/garçons est presque atteinte à l'école primaire, mais le pourcentage baisse ensuite, surtout dans le domaine des sciences et de la formation scientifique et technique. Moins de $5 \%$

\section{Refonder l'éducation avec les médias : l'exemple de la médiatisation du programme "Vacances citoyennes"}

L'université Cheikh Anta Diop de Dakar a mis en place ce programme qui vise à développer le savoir-être des étudiants. Il s'agit d'envoyer les étudiants dans les terroirs, pour donner des services, en l'occurrence le reboisement, la campagne sanitaire, l'alphabétisation dans les langues nationales et l'initiation à l'informatique. Ce programme mobilise cinquante étudiants par camp, durant quinze jours pendant les vacances. Il est effectué en partenariat avec les ministères concernés par les services, mais aussi les entreprises, dans le cadre de leur responsabilité sociale. En somme, l'université mobilise les étudiants et leurs encadrants, ainsi que des moyens logistiques, et les partenaires mobilisent les ressources financières.

Dans ce cas précis, les médias ont bien couvert les cérémonies de lancement et de clôture. Les activités des étudiants étaient quotidiennement relayées par la presse. Cela tient au fait que les étudiants du CESTI, l'école sénégalaise de formation en journalisme, étaient disséminés dans les différents camps. Ces étudiants donnaient les informations à la presse et assez souvent, ils y étaient des pigistes ou des stagiaires. Ces étudiants du CESTI ont même créé un blog dans leur établissement, qui hébergeait les résultats des camps citoyens, ce qui fait que les étudiants décrivaient la situation des terroirs à l'intérieur et à l'extérieur du pays mais donnaient aussi les résultats auxquels les étudiants parvenaient sur le terrain.

Cela a permis aux populations de percevoir l'enseignement supérieur sous un autre angle : celui des services rendus à la communauté. Il faut dire que la situation de l'éducation au Sénégal est très différente de celle des sociétés occidentales, où elle correspond à une évolution de la société. Au Sénégal, l'éducation actuelle est un greffon de la colonisation qui n'a pas suffisamment été questionné, à l'indépendance, pas suffisamment internalisé, afin qu'il serve notre propre vision. C'est tout le travail qui est mené actuellement pour que ce soit diffusé auprès des communautés, et les camps citoyens devaient répondre à cet impératif.

Beaucoup de Sénégalais ne connaissent pas l'université ou seulement de loin. Ils n'en ont connaissance que lorsque la presse en parle... et la presse n'en parle que lorsqu'il il y a des grèves. Tous les bienfaits, tous ceux que l'université forme et qui occupent des positions très élevées dans la société, qui s'en sortent par l'éducation, ce n'est pas aussi bien diffusé ou connu. Donc cette opération d'information, de communication, qui vise à aller vers la société et à refonder le système éducatif en y raccordant l'ensemble des valeurs que le pays a et projette dans le futur, c'est un champ énorme qui fonde aujourd'hui aussi les Assises. 
des apprenants bénéficient d'une formation technique et professionnelle. Les apprentissages se font encore sur le tas. Les langues maternelles tardent à être généralisées dans le système éducatif. Malheureusement, il y a encore $57 \%$ d'analphabètes au Sénégal, et une pauvreté difficile qui empêche de dépenser pour l'achat de journaux ou de livres. Combien de Sénégalais ont des ordinateurs, combien ont accès à Internet?

Il est impossible, dans cette situation, de pouvoir envisager le développement du Sénégal dans un monde fondé de plus en plus sur le savoir. C’est la raison d'être des Assises de l'éducation. Les efforts financiers très importants consentis par le gouvernement ces dernières années en matière d'éducation sont torpillés par des grèves répétitives d'enseignants. C'est pourquoi les autorités politiques nous ont demandé de réfléchir à une refonte d'ensemble du système éducatif.

Trois comités structurent cette instance : le comité national de pilotage, le comité d'appui scientifique et le comité d'organisation. Toutes les quatorze régions ont produit les rapports de leur concertation, ainsi que les sept commissions thématiques (www.assises-education.sn). Les Assises de l'éducation font émerger un ressenti largement partagé : l'école publique a aujourd'hui d'énormes difficultés. De nombreux Sénégalais inscrivent leurs enfants dans le privé, notamment l'enseignement privé catholique, même si un certain nombre d'enseignements privés islamiques commencent à se développer. Ces établissements emploient aussi des enseignants du public. Et en effet avec des infrastructures, une bonne gestion des effectifs, des élèves inscrits et certains enseignants du public, on obtient des performances. Donc, la réflexion des Assises porte plus sur l'enseignement public, qui doit retrouver sa performance d'antan.

\section{QUELQUeS PISTES POUR AMÉLIORER LA QUALITÉ DES MÉDIAS EN ÉDUCATION}

Nous avons très tôt mis l'accent sur la place et le rôle de la presse dans un système éducatif. L'éducation, ce n'est pas l'école seulement, c'est aussi l'environnement de l'école et les familles. Nous avons organisé une rencontre avec les patrons de presse pour les sensibiliser au rôle de la presse dans l'éducation, et en appeler à leur co-responsabilité. Nous souhaitions attirer leur attention sur la faible qualité du traitement actuel de l'éducation dans les médias sénégalais, voire son absence. C'est aussi une question de marché. Avec un événement sensationnel, les ventes sont élevées. Mais avec des articles de fond, sérieux, les ventes sont très faibles. Or, les propriétaires des maisons d'édition ou de presse sont des marchands. Certains n'ont même aucune qualification journalistique!

Nous voulons, avec le Comité national de régulation de l'audiovisuel (CNRA), créer un observatoire des pratiques éducatives des médias, avec l'espoir d'aboutir à un ranking des meilleurs médias. Notre objectif est d'amener la population à s'intéresser progressivement à ces médias plutôt qu'à ceux qui ne 
font que de l'événementiel. Nous avons obtenu l'appui de l'Agence de régulation des médias et des organisations des journalistes, et cette idée fera l'objet d'une recommandation au gouvernement des Assises de l'éducation, dont le rôle est de formuler des stratégies.

Ces transformations ne se feront pas du jour au lendemain mais dans la durée : dès lors que les rédactions seront tenues d'avoir des rubriques éducation, de produire sur l'éducation, elles devront s'informer. En outre, aux Assises, nous souhaitons aider les journalistes à renforcer leurs compétences pour traiter du système éducatif, afin qu'ils connaissent mieux les éléments qui déterminent la performance éducative. Notre projet est de construire un système cohérent. Tout cela devra se mettre en place dans la durée.

Nous soumettrons nos recommandations au président de la République, qui décidera en conseil s'il les retient et s'il les traduit en directives, qui deviendront alors opératoires.

\section{Un exemple de radio communautaire}

Mon projet de créer une radio communautaire et d'y intégrer des sujets d'éducation est lié à mon histoire personnelle. Ma mère était originaire d'une ville au nord du pays. Avant sa mort, elle a souhaité que sa famille retourne aider les populations locales. Comme ce que je sais faire, c'est partager la connaissance, j'ai proposé de créer un centre qui serait une interface entre les universités et les communautés. Nous avons financé la construction de ce centre sur un terrain qui appartient à mon père et, lorsque j'ai réfléchi à ce qu'il était possible de faire pour aider la communauté, je me suis rendu compte qu'on pouvait installer sur le site un centre communautaire multimédias, avec une salle informatique et une radio communautaire, pour lesquelles j'ai pu trouver des financements. En ce qui concerne les programmes de cette radio, j'ai proposé des émissions animées par les élèves pour les élèves, à tous les niveaux - primaire, moyen, secondaire, technique - et en français. Pour l'essentiel, cette radio vise le développement. Dans son comité de pilotage, tous les corps sociaux sont représentés. Et chaque corps social devra animer une émission.

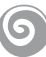

Le rôle de la presse dans le fonctionnement de l'école et la gouvernance de l'éducation est capital. Si la presse commence à publier des articles sur les performances des établissements, sur les éléments de fond, sur les comportements, etc., la société sera mieux informée et réservera une place importante à l'éducation. Cela ne concerne pas ceux qui sont instruits, et qui investissent déjà des sommes énormes dans l'éducation de leurs enfants. Mais dans un contexte de grande pauvreté au Sénégal, il est essentiel de diffuser l'idée que chacun doit investir dans l'éducation et en retirera des bénéfices, et la presse devra nous y aider. Je suis convaincu que si tout le monde comprend les bienfaits de l'éducation, la presse saura accompagner ce processus. 